\title{
Evidence of a systemic phenomenon for oxidative stress in cholestatic liver disease
}

P Ljubuncic, Z Tanne, A Bomzon

\begin{abstract}
Background-There is considerable evidence indicating that the severity of hepatic damage in individuals with cholestatic liver disease is causally associated with the extent of intrahepatic oxidative stress. Increased levels or accelerated generation of reactive oxygen species and toxic degradative products of lipid peroxidation have been reported in the plasma of individuals with chronic liver disease and animal models of liver disease. Hence, by virtue of their increased presence in the circulation, it is not unreasonable to suppose that they may account for extrahepatic tissue damage in chronic liver disease.
\end{abstract}

Materials and methods-This hypothesis was tested by determining plasma levels of the ubiquitous antioxidant glutathione (GSH) and lipid peroxides (LP), together with assessment of the extent of lipid peroxidation in the kidney, brain, and heart, in 24 day chronically bile duct ligated (CBDL) rats. The extent of lipid peroxidation in tissues was based on measurement of conjugated dienes, lipid peroxides, and malondialdehyde (MDA) content. Data were compared with identical data collected from unoperated control, pair fed, 24 day bile duct manipulated (sham operated), and pair fed sham operated rats.

Results-In CBDL rats, total and reduced plasma GSH levels were almost half those determined in all control rats. Plasma, kidney, and heart LP levels were significantly increased in CBDL rats compared with controls. MDA levels were significantly higher in the kidney, brain, and heart homogenates prepared from CBDL rats compared with MDA content measured in tissue homogenates prepared from the four groups of control rats.

Conclusions-Our data show that experimental cholestatic liver disease is associated with increased lipid peroxidation in the kidney, brain, and heart. Hence we have concluded that the oxidative stress in cholestatic liver disease is a systemic phenomenon probably encompassing all tissues and organs, even those separated by the blood-brain barrier.

(Gut 2000;47:710-716)

Keywords: cirrhosis; oxidative stress; lipid peroxidation; lipid peroxides; conjugated dienes; malondialdehyde; glutathione

There is evidence that the diseased liver of patients with cholestatic liver disease is exposed to oxidative stress associated with increased lipid peroxidation. ${ }^{12}$ Oxidative stress and increased lipid peroxidation are also present in the kidney of animal models of cholestatic liver disease (for review see Bomzon and colleagues $^{3}$ ). The underlying basis of oxidative stress in the liver and kidney is complex, involving intraorgan generation of reactive oxygen species (ROS), possibly mediated by endotoxin ${ }^{4}$ and bile acids, ${ }^{35}$ and accumulation of degradative products of lipid peroxidation, such as lipid peroxides (LP) and/or malondialdehyde (MDA). ${ }^{6}$ Increased levels or accelerated generation of ROS, such as superoxide anion and hydroxyl radical, have been reported in the plasma of patients with liver disease and animal models of liver disease. ${ }^{78}$ Elevated plasma levels of LP have been reported in patients with liver disease. ${ }^{9}$ The increased presence of ROS and these toxic products in plasma raises the possibility that they could exert their injurious actions in the kidney and other extrahepatic tissues, as well as in the diseased liver.

We have addressed this possibility by measuring the extent of lipid peroxidation in the heart and brain of the 24 day chronic bile duct ligated rat (CBDL), a widely used animal model of cholestatic liver disease. ${ }^{10}$ Increased lipid peroxidation in the liver and kidney in this animal model of cholestatic liver disease has been extensively reported. ${ }^{11-13}$ The extent of lipid peroxidation was based on determination of conjugated dienes (CD), LP, and MDA in the brain and heart. For comparison purposes, we sought confirmation of increased lipid peroxidation in the liver and kidney using identical biochemical indices, as well as measuring plasma levels of the ubiquitous intracellular antioxidant glutathione (GSH) and LP in this animal model of cholestatic liver disease. Data were compared with identical data from unoperated control, 24 day pair fed (PF), 24 day bile duct manipulated (sham operated (SO)), and 24 day PF SO rats. Consistent with the internationally accepted principle that a minimum number of animals be used to achieve the objectives of a study involving experimental animals, data on body, liver, and spleen weights and plasma indices of hepatic damage, together with the extent of lipid peroxidation in the liver, were taken from a soon to be published paper involving identical animals. ${ }^{14}$

Abbreviations used in this paper: $\mathrm{BDL} / \mathrm{CBDL}$, (chronic) bile duct ligated/ligation; $\mathrm{CD}$, conjugated dienes; GSH, glutathione; GSSG, oxidised glutathione; LP, lipid peroxides; MDA, malondialdehyde; PF, pair fed; ROS, reactive oxygen species; $\mathrm{SO}$, sham operated; TBARS, thiobarbituric acid reactive substances. 
Materials and methods

ANIMAL MODEL

Fifty four male Sprague-Dawley rats, weighing 300-350 g, were used in the experiments. The surgical procedures and experimental protocols were approved by the Technion Committee for Supervision of Animal Experiments. Rats were housed in a temperature and humidity controlled holding facility with a 12:12 hour light-dark cycle. The rats had free access to chow and water. The present study was undertaken in CBDL rats. This rat model of cholestatic liver disease and secondary biliary cirrhosis has been used previously in our laboratory. ${ }^{15} 16$ Under ketamine anaesthesia (120 $\mathrm{mg} / \mathrm{kg}$ intraperitoneally), the common bile duct was exposed through a midline abdominal incision in 16 rats. It was ligated in two places with 4-0 silk thread and sectioned between the ligatures. After surgery, all rats received ampicillin (30000 units intramuscularly) and vitamin $\mathrm{K}$ (1 mg intramuscularly; Konakion, Roche) and thereafter weekly until completion of the experiment.

Five CBDL rats died postoperatively. Twenty four days following bile duct ligation, the remaining 11 rats were anaesthetised with pentobarbitone sodium $(35 \mathrm{mg} / \mathrm{kg})$. The abdomen was opened and the lower abdominal aorta exposed. Approximately $10 \mathrm{ml}$ of arterial blood were withdrawn through an aortic 21 gauge needle puncture for measurement of plasma GSH and LP concentrations. The kidneys, heart, and brain were harvested for determination of the extent of lipid peroxidation (see below for details). Individual plasma samples and organs were numerically identified and stored at $-70^{\circ} \mathrm{C}$ until required. Data on plasma GSH levels and extent of lipid peroxidation in kidneys from SO rats could not be obtained due to refrigeration failure resulting in irretrievable sample loss.

Identical plasma and tissue collection procedures were performed in 17 control, seven PF, seven SO, and seven PF SO rats whose bile ducts had been exposed and manipulated 24 days previously. PF animals were prepared by restricting their food intake over 24 days and daily weighing to affect a $10 \%$ decrease in individual body weight after 24 days. Four control groups were used in this study for two reasons. Firstly, we have previously shown that the sham operation procedure may influence in vitro study parameters. ${ }^{17}$ Secondly, BDL rats are malnourished and do not thrive, ${ }^{14} 19$ and malnutrition has been reported to enhance the extent of lipid peroxidation. ${ }^{20}{ }^{21}$ Hence incorporation of four control groups into the experimental design was necessary to avoid these confounding influences.

PREPARATION OF CRUDE TISSUE HOMOGENATES On the day of quantification, randomly chosen kidneys and brains from each group were thawed, freed of unnecessary tissue, cut into small pieces by fine scissors, and homogenised in ice cold $100 \mathrm{mM}$ sodium phosphate buffer, $\mathrm{pH} 7.4$, at $4^{\circ} \mathrm{C}$ using a Potter-Elvehjem glass homogeniser. Crude homogenates of thawed hearts (also cleaned and cut into small pieces) were prepared in identical buffer using a Polytron homogeniser by three five second bursts, set at 7 . Separate aliquots of each homogenate were used for CD, LP, and TBARS assays (see below for details). Protein content of individual tissue homogenates was determined using the method of Lowry and colleagues, ${ }^{22}$ with crystalline bovine serum albumin (Sigma Chemical Corp, St Louis, Missouri, USA) as standard.

\section{ANALYTICAL PROCEDURES}

Determination of plasma GSH levels

Determination of plasma GSH levels using GSH reductase was performed according to the method of Adams and colleagues ${ }^{23}$ with immediate acidification of plasma after blood centrifugation (3000 rpm for five minutes) to prevent spontaneous oxidation of GSH $(50 \mu \mathrm{l}$ of 50\% 5-sulphosalicyclic acid/1 ml plasma). The acidified plasma samples were frozen at $-70^{\circ} \mathrm{C}$ for a maximum of three weeks. Before measurement of GSH, the sample was thawed and back titrated to $\mathrm{pH} 7$ with $0.2 \mathrm{~mol} / \mathrm{l}$ $\mathrm{NaOH}$. Two separate measurements were performed: total plasma GSH and the oxidised form (GSSG). The reduced plasma GSH level was calculated as the difference between total GSH and the oxidised form. Total plasma GSH concentration was determined using $10 \mathrm{mmol} / 1$ 5,5'-dithio-bis-2-nitrobenzoic acid, after reduction of GSSG by GSSG reductase (derived from Baker's yeast, Sigma Chemical Corp). The resulting amount of $\mathrm{GSH}$ was determined from a standard curve $(0-2.5 \mathrm{nmol}$ GSSG), measured spectrophotometrically at $412 \mathrm{~nm}$ and expressed as change in absorbance per minute during reduction with GSH reductase. To measure plasma GSSG content, alkylation of the $\mathrm{SH}$ groups of glutathione was carried out with freshly prepared $10 \mathrm{mmol} / \mathrm{l}$ $\mathrm{N}$-ethylmaleimide. To avoid the adverse effects of excess $N$-ethylmaleimide on the enzyme reaction used to determine GSSG levels, excess $\mathrm{N}$-ethylmaleimide was removed by separation on a Sep-Pak (C18) column (Sigma Chemical Corp).

\section{Determination of plasma LP content}

Plasma LP content was determined using a commercially available kit purchased from Kamiya Biochemical Co. (Washington, USA). The assay is based on the observation that haemoglobin catalyses a reaction between LP and a methylene blue derived chromogen, 10-N-methylcarbamoyl-3,7-dimethylamino$10 \mathrm{H}$-phenothiazine forming an equimolar concentration of methylene blue with maximum absorbance at $675 \mathrm{~nm}$. Duplicate determinations in $100 \mu \mathrm{l}$ plasma samples collected from all animals were made and the average of two measurements was used in subsequent statistical analysis of the data.

\section{Determination of tissue CD content}

The formation of CD is one of the early biochemical events associated with the process of lipid peroxidation. ${ }^{6}$ They arise when ROS attack hydrogen atoms of methylene groups separating double bonds in polyunsaturated 
Table 1 Plasma levels of glutathione (GSH, its redox state, and its oxidised form (GSSG)) in control, pair fed (PF), pair fed sham operated (PF SO), and cirrhotic (CBDL) rats. No SO data are reported (see text for details).

\begin{tabular}{llllll}
\hline & Control $(n=17)$ & PF $(n=7)$ & PF SO $(n=7)$ & \multicolumn{2}{l}{ CBDL $(n=11)$} \\
\hline Total GSH $(\mu \mathrm{M})$ & $9.52(1.09)$ & $11.52(1.98)$ & $10.04(1.82)$ & $4.22(0.54)$ & $<0.005$ \\
GSSG $(\mu \mathrm{M})$ & $2.42(0.38)$ & $2.04(0.36)$ & $2.53(0.48)$ & $0.76(0.16)$ & $\mathrm{ns}$ \\
Reduced GSH $(\mu \mathrm{M})$ & $7.10(1.07)$ & $9.48(1.77)$ & $7.52(1.66)$ & $3.48(0.47)$ & $<0.005$ \\
GSH redox state $(\mathrm{GSSG} / \mathrm{GSH})$ & $0.26(0.04)$ & $0.19(0.03)$ & $0.27(0.04)$ & $0.22(0.05)$ & $\mathrm{ns}$ \\
\hline
\end{tabular}

Data are mean (SEM).

Significance values represent differences between the CBDL group and the three control groups (control, PF, and PF SO).

fatty acids. ${ }^{6}$ Their presence in tissue homogenates reflects their formation and indicates the initiation of the lipid peroxidation process. Determination of CD in the kidney, brain, and heart homogenates was identical to that described by Fuhrman and colleagues. ${ }^{24}$ An aliquot $(1 \mathrm{ml})$ of tissue homogenate underwent hexane:isopropanol $(3: 2, \mathrm{v} / \mathrm{v})$ lipid extraction followed by evaporation of the upper (hexane) phase by an oxygen free nitrogen stream in a light protected environment to quantify the tissue contents of CD and LP. For CD determinations, the sample was redissolved in $1 \mathrm{ml}$ of hexane. Tissue CD content was detected spectrophotometrically at an optical density of $234 \mathrm{~nm}$ against a hexane blank. ${ }^{25}$

Determination of tissue LP content

The presence of LP in biological samples is indicative of propagation of the lipid peroxidation process. ${ }^{25} \mathrm{LP}$ content of the tissue samples was determined using the method of El-Saadani and colleagues ${ }^{26}$ which uses the cholesterol colour reagent of a commercially available kit to measure cholesterol (Diagnostica Merck, Darmstadt, Germany). The basis of the determination relies upon the ability of LP to convert iodide to iodine. After lipid extraction and drying under an oxygen free nitrogen stream in a light protected environment, $1 \mathrm{ml}$ of colour reagent was added to the sample and incubated at room temperature for 30 minutes in the dark. On completion of the incubation, LP content was determined by recording absorbance at $365 \mathrm{~nm}$ against the reagent blank.

Determination of tissue MDA content

MDA is an end product of peroxidative decomposition of polyenic fatty acids in the lipid peroxidation process and its accumulation in tissues is indicative of the extent of lipid peroxidation. ${ }^{6}$ Tissue MDA was measured using the thiobarbituric acid reactive substances (TBARS) assay, as described by Draper and Hadley. ${ }^{27}$ TBARS reagent $(1 \mathrm{ml})$ was added to a $0.5 \mathrm{ml}$ aliquot of tissue homogenate and heated for 20 minutes at $100^{\circ} \mathrm{C}$. The antioxidant, butylated hydroxytoluene, was added before heating the samples. After cooling on ice, samples were centrifuged at $840 \mathrm{~g}$ for 15 minutes and absorbance of the supernatant was read at $532 \mathrm{~nm}$. Blanks for each sample were prepared and assessed in the same way to correct for the contribution of A532 to the sample. TBARS results were expressed as MDA equivalents using 1,1,3,3tetraethoxypropane as standard.
STATISTICAL ANALYSIS

For each study parameter, measurements were made in duplicate and the average of the two measurements was used in subsequent statistical analysis. Data were analysed by one way analysis of variance (ANOVA) with a Bonferroni post test. Data are expressed as mean (SEM). p $<0.05$ (two tailed) was considered statistically significant.

\section{Results}

Compared with all four control groups, CBDL rats had elevated plasma activities of alanine and aspartate transferase, alkaline phosphatase, and raised plasma levels of bilirubin and cholesterol. Histology of liver biopsies verified the presence of secondary biliary

\begin{tabular}{l}
$\square$ Control rats $\quad \square$ Pair fed sham operated rats \\
$\square$ Pair fed rats $\quad \square$ Cirrhotic (CBDL) rats \\
\hline
\end{tabular}
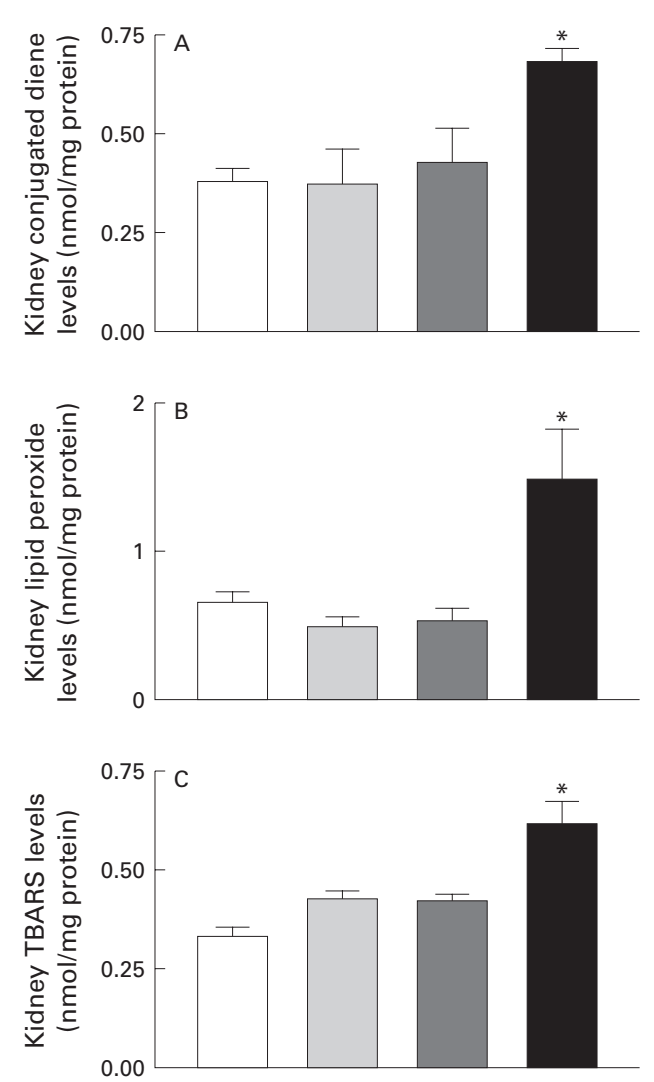

Figure 1 Extent of lipid peroxidation, measured as content of conjugated dienes $(A)$, lipid peroxides (B), and malondialdehyde (TBARS) (C) in kidney homogenates prepared from control, pair fed, pair fed sham operated, and chronic bile duct ligated $(C B D L)$ rats $(n=7-17)$. (There were no data on sham operated rats (see text for details). ${ }^{{ }^{*}} p<0.05$ compared with the three control groups. 

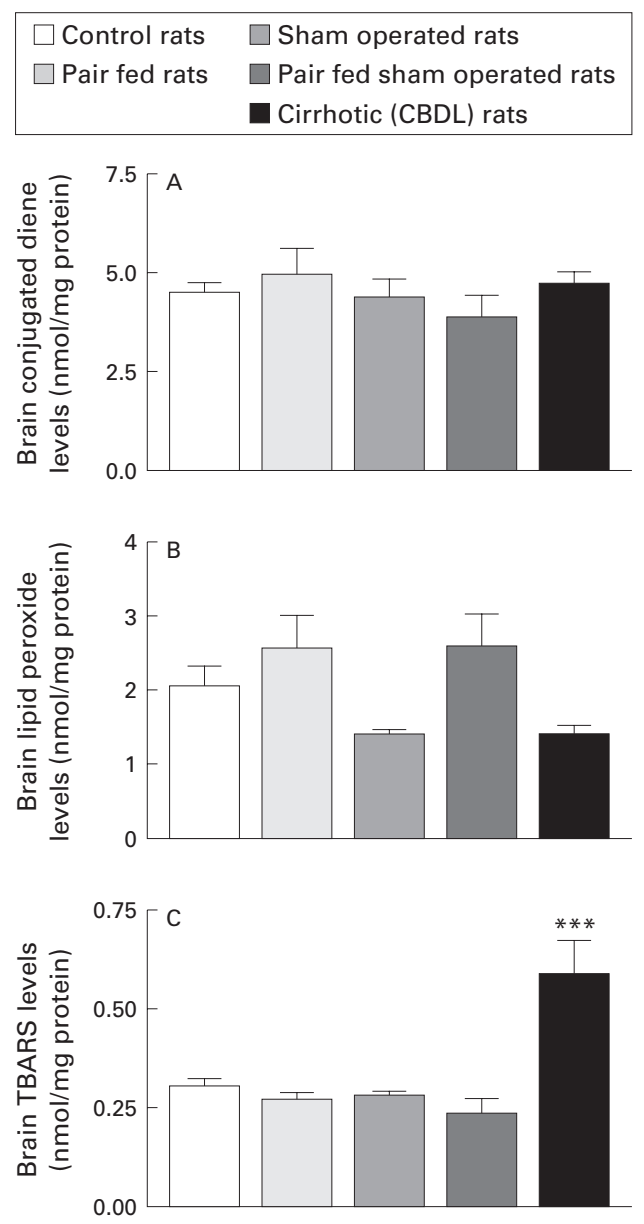

Figure 2 Extent of lipid peroxidation, measured as content of conjugated dienes $(A)$, lipid peroxides (B), and malondialdehyde (TBARS) (C) in brain homogenates prepared from control, pair fed, sham operated, pair fed sham operated, and chronic bile duct ligated (CBDL) rats $(n=7-17) .{ }^{\star *} p<0.001$ compared with the four control groups.

cirrhosis. In addition, the livers of these rats had an elevated MDA content indicative of increased hepatic lipid peroxidation (data not shown).

PLASMA GLUTATHIONE LEVELS

In CBDL rats, total GSH levels were approximately half those determined in control rats (table 1). This decrease was associated with a significant reduction in plasma levels of reduced GSH in CBDL rats compared with control values (table 1). There was a tendency for the glutathione redox state to increase in CBDL rats (table 1). In addition, there were no significant changes in GSSG levels in CBDL rats compared with control rats (table 1). We were unable to detect statistically significant correlations between changes in individual plasma GSH study parameters and individual plasma indices of hepatic damage.

PLASMA LIPID PEROXIDE CONTENT

In control rats, there were no detectable plasma levels of LP. However, LP were detected in the plasma of CBDL rats $(0.52(0.14) \mathrm{nmol} / \mathrm{ml})$.

\begin{tabular}{|ll|}
\hline Control rats & $\square$ Sham operated rats \\
$\square$ Pair fed rats & $\square$ Pair fed sham operated rats \\
& $\square$ Cirrhotic (CBDL) rats
\end{tabular}
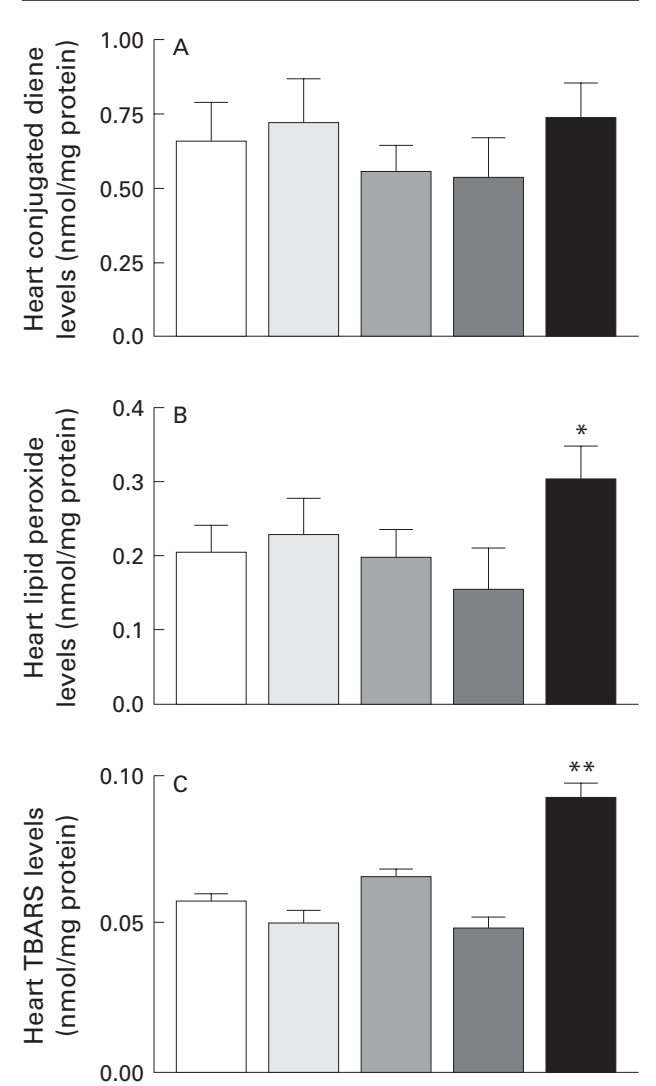

Figure 3 Extent of lipid peroxidation, measured as content of conjugated dienes $(A)$, lipid peroxides $(B)$, and malondialdehyde (TBARS) (C) in heart homogenates prepared from control, pair fed, sham operated, pair fed sham operated, and chronic bile duct ligated (CBDL) rats $(n=7-17) .{ }^{*} p<0.05,{ }^{\star} *<<0.01$ compared with the four control groups.

TISSUE INDICES OF LIPID PEROXIDATION

Kidney

CD, LP, and MDA levels were significantly greater $(p<0.05)$ in kidney homogenates prepared from CBDL rats than those measured in kidney homogenates from control rats (fig 1). Furthermore, there were no significant differences between levels of these indices between the four groups of control rats.

Brain

CD and LP levels in brain homogenates prepared from control and CBDL rats were not significantly different (fig 2). In contrast, the MDA content in brain homogenates prepared from CBDL rats was twice $(p<0.001)$ that of levels found in brain homogenates prepared from all groups of control rats (fig 2). In addition, MDA levels were not significantly different between the four control groups (control, $\mathrm{PF}, \mathrm{SO}$, and PF SO rats).

Heart

There were no significant differences in CD levels in heart homogenates prepared from control and CBDL rats (fig 3). In CBDL rats, tissue LP content was significantly elevated 
$(\mathrm{p}<0.05)$ compared with control rats (fig 3). Consistent with that described in the other tissues, MDA content in heart homogenates prepared from the CBDL rats was significantly elevated $(p<0.01)$ compared with levels determined in homogenates from all groups of control rats (fig 3).

\section{Discussion}

One method of detecting oxidative stress is to estimate the ratio between plasma or tissue levels of endogenous antioxidants, such as GSH, and indices of the extent of lipid peroxidation, such as LP or MDA. ${ }^{28} \mathrm{We}^{14}$ and others ${ }^{11} 1829$ have demonstrated increased lipid peroxidation in the diseased liver of CBDL rats. In the present study, we found reduced levels of GSH and elevated LP content in plasma of CBDL rats compared with controls. Furthermore, we detected increased lipid peroxidation in the kidneys, brain, and heart in identical CBDL rats. Therefore, we conclude that oxidative stress of cholestatic liver disease is not restricted to the diseased liver and can be considered a systemic phenomenon.

Before discussing the data, we wish to comment on the presence of elevated MDA levels in the kidneys, brain, and heart with variable changes in individual tissue levels of CD and $\mathrm{LP}$ in 24 day CBDL rats. The presence of CD, $\mathrm{LP}$, and MDA is indicative of different phases of the lipid peroxidation process in which CD and LP are intermediatory products while MDA is one of the accumulated end products. $^{28}$ Detection of MDA using the TBARS assay is widely used to detect the presence of the lipid peroxidation process and ROS activity. ${ }^{6} 280$ In cholestatic liver disease, the membrane phospholipid profile and/or composition is abnormal ${ }^{31}$ and content is depleted. ${ }^{32}$ In tissues with relatively low phospholipid content such as the heart, further shifts in phospholipid content or composition due to ROS attack become magnified. Thus if absolute values of MDA in the various tissues harvested from CBDL rats are compared, it is not surprising that the lowest values are found in heart homogenates and the greatest values in brain homogenates. To interpret the absence of changes in tissue levels of CD or LP as being indicative of the absence of oxidative stress is a reasonable conclusion when no increases in MDA levels are seen in the same tissue. Our demonstration of consistent significant increases in MDA content of tissue homogenates prepared from CBDL rats is indicative of increased activity of the lipid peroxidation process. Moreover, we do not consider these reproducible increases as repetitive type 1 errors.

During the 1980 s, isolated communications began to appear in the literature reporting increased plasma content of markers of lipid peroxidation, such as LP, in liver disease. ${ }^{33}$ Since the publication of these studies, there have been many reports confirming the presence of oxidative stress in liver disease in humans and experimental animals using a variety of plasma markers. ${ }^{13435}$ In liver disease, GSH levels are depleted due to reduced production. ${ }^{36}{ }^{37}$ Plasma GSH levels reflect intracellular levels. ${ }^{23}$ In healthy individuals, the plasma content of LP is negligible and their presence in plasma is indicative of an ongoing oxidative process. ${ }^{38}{ }^{39}$ Therefore, our data on reduced plasma GSH levels and increased plasma content of LP in the CBDL rat are consistent with these earlier observations that cholestatic liver disease is associated with a noticeable reduction in the availability of an important endogenous antioxidant and the presence of an ongoing oxidative process.

Using identical biochemical markers as those used to describe increased lipid peroxidation in the diseased liver of CBDL rats,${ }^{14}$ we also observed increased lipid peroxidation in the kidneys, brain, and heart of CBDL rats. The presence of oxidative stress in the brain and heart of CBDL rats has not been previously reported. This finding could have been anticipated by virtue of enhanced ROS generation and the presence of degradative products of lipid peroxidation in the plasma of individuals and animals with liver disease. ${ }^{79}$ Although we did not seek evidence of oxidative stress in other organs, such as the intestine, lung, and reproductive organs, we can only speculate that increased lipid peroxidation will probably be found in other extrahepatic tissues.

The main new findings of our study were enhanced lipid peroxidation in the heart and brain of BDL rats. Following BDL, these rats do not thrive, are anorexic, and lose weight. $^{141819}$ It has also been reported that starvation of three and seven days' duration can enhance the extent of lipid peroxidation in the brain $^{21}$ and heart, ${ }^{20}$ respectively. To eliminate the possibility that the nutritional status of the BDL rats did not confound our data, we incorporated two groups of pair fed animals (PF group and PF SO group). The extent of lipid peroxidation in the heart and brain, as well as in the liver and kidneys, in these two groups of $\mathrm{PF}$ animals was similar to that determined in the control and SO rats. Therefore, we conclude that the presence of increased lipid peroxidation in the tissues of cirrhotic (CBDL) rats was a direct consequence of ligation of the bile duct for 24 day and was not caused by alterations in nutritional risk factors.

What triggers the onset of oxidative stress in extrahepatic tissues in cholestatic liver disease? Chronic activation of the sympathetic and renin-angiotensin systems, reflected by increases in plasma concentrations of catecholamines and angiotensin II, occurs in chronic liver disease. ${ }^{40}$ Catecholamines are prooxidants stimulating generation of ROS. ${ }^{41}$ Angiotensin II can generate superoxide anion release by stimulating an NADH/NADPH dependent, membrane bound oxidase present in the membranes of vascular smooth muscle and endothelium, ${ }^{42}$ as well as circulating neutrophils. ${ }^{43}$ Bile acids accumulate in the diseased liver and plasma of patients with cholestatic liver diseases. ${ }^{44}$ In CBDL rats, serum bile acid levels increase 80-100 fold, with urinary excretion exceeding normal biliary secretion. ${ }^{32}$ Bile acids are pro-oxidants causing direct tissue damage mediated by $\operatorname{ROS}^{54}$ or indirectly 
through activation of tissue resident macrophages to release $\operatorname{ROS}^{46}$ It is tempting to speculate that their increased presence in plasma and kidney could trigger the onset of the lipid peroxidation process as well as account for the difference in the patterns of lipid peroxidation in the kidney and heart compared with the brain after 24 days of bile duct ligation. Bile acids can also accumulate intracellularly by virtue of their ability to bind to plasma lipoproteins, specifically low density lipoprotein, ${ }^{47}$ that are targeted to all cells. Thus low density lipoproteins may play a major role in the transport of potentially toxic bile acids from plasma into the intracellular milieu of extrahepatic tissues to generate ROS when the integrity of the enterohepatic circulation is disrupted due to portosystemic shunts and/or bile acid spillover into the systemic circulation. Furthermore, plasma MDA and similar substances aggravate ROS induced cytotoxicity. ${ }^{6}$ Hence the oxidative stress in extrahepatic tissues in cholestatic liver disease may be linked to chronic exposure of tissues to elevated plasma concentrations of catecholamines, angiotensin, hydrophobic bile acids, and the degradative products of lipid peroxidation.

Is there a causal relationship between oxidative stress and organ malfunction in liver disease? Such a causal relationship has been reported in the diseased liver derived from evidence reporting a beneficial therapeutic response of antioxidants in animal models of liver disease. ${ }^{48}$ This relationship has been extended to extrahepatic organs or systems where complications exist in chronic liver disease, such as the kidney ${ }^{49}$ and the hyperdynamic circulation. ${ }^{50}$ In view of these relationships, can our data on increased lipid peroxidation in the brain and heart be implicated in the pathogenesis of hepatic cardiomyopathy or encephalopathy? A causal relationship between oxidative stress and loss of cardiac function has been proposed as the basis of cardiomyopathy in cardiac failure. ${ }^{51}$ Although cardiomyopathy, with features similar to that of cardiac failure, has been described in CBDL rats, ${ }^{52}$ we were unable to find any reports linking oxidative stress to hepatic cardiomyopathy. Moreover, oxidative stress has not been implicated as a contributory component in the development of hepatic encephalopathy. ${ }^{53}$ While there is no dispute linking increased generation of ROS to cell/tissue/organ damage, additional studies are required to establish whether there are causal relationships between oxidative stress and other extrahepatic complications of liver disease.

In conclusion, our study showed that experimental cholestatic liver disease was associated with: increased lipid peroxidation in the brain and heart, in addition to increased presence in the liver and kidney; and depleted plasma levels of the ubiquitous antioxidant glutathione in conjunction with elevated plasma LP content. These data indicate that oxidative stress in cholestatic liver disease is a systemic phenomenon, probably encompassing all tissues and organs, even those separated by the bloodbrain barrier.
This study was supported by grants from the Center for Absorption in Science, Ministry of Immigration and Absorption, Jerusalem, Israel (PL), the Chief Scientist, Ministry of Health, Jerusalem, Israel (AB), and the Technion Vice-President for Research, Technion-IIT, Haifa, Israel (AB).

1 Tsai LY, Lee KT, Tsai SM, et al. Changes of lipid peroxide evels in blood and liver tissue of patients with obstructive aundice. Clin Chim Acta 1993;215:41-50.

2 Ono M, Sekiya C, Ohhira M, et al. Elevated level of serum $\mathrm{Mn}$-superoxide dismutase in patients with primary biliary cirrhosis: possible involvement of free radicals in the pathogenesis in primary biliary cirrhosis. I Lab Clin Med 1997;118:476-83.

3 Bomzon A, Holt S, Moore K. Bile acids, oxidative stress, and renal function in biliary obstruction. Semin Nephrol 1997; 17:549-62.

4 Bailey ME. Endotoxin, bile salts and renal function in obstructive jaundice. Br F Surg 1976;63:774-8.

5 Sokol RJ, Devereaux M, Khandwala R, et al. Evidence for involvement of oxygen free radicals in bile acid toxicity to isolated rat hepatocytes. Hepatology 1993;17:869-81.

6 Halliwell B. Reactive oxygen species in living systems: source, biochemistry, and role in human disease. Am $\mathrm{f} \mathrm{Med}$ 1991;91(suppl 3C):14-22S.

7 Chen MF, Mo LR, Lin RC, et al. Increase of resting levels of superoxide anion in the whole blood of patients with decompensated liver cirrhosis. Free Radic Biol Med 1997;23:672-9.

8 Tsai LY, Lee KT, Liu TZ. Evidence for accelerated generation of hydroxyl radicals in experimental obstructive jaundice of rats. Free Radic Biol Med 1998;24:732-7.

9 Fabris C, Pirisi M, Panozzo MP, et al. Intensity of nflammatory damage and serum lipid peroxide concentrations in liver disease. 7 Clin Pathol 1993;46:364-7.

10 Bomzon A, Blendis LM. Animal models of liver disease. In: Bomzon A, Blendis LM, eds. Cardiovascular complications of liver disease. Boca Raton: CRC Press, 1990:9-28.

11 Singh S, Shackleton G, Ah-Sing E, et al. Antioxidant defenses in the bile duct-ligated rat. Gastroenterology 1992; 103:1625-9.

12 Muriel P, Suarez OR. Role of lipid peroxidation in biliary obstruction in the rat. F Appl Toxicol 1994;14:423-6.

13 Paradis V, Kollinger M, Fabre M, et al. In situ detection of lipid peroxidation by-products in chronic liver diseases. Hepatology 1997;26:135-42.

14 Ljubuncic P, Tanne Z, Bomzon A. Ursodeoxycholic acid suppresses the increased extent of lipid peroxidation in the diseased liver in experimental cholestatic liver disease. Dig Dis Sci 2000 (in press)

15 Bomzon A, Jacob G, Lee SS, et al. In vitro vascular responsiveness to norepinephrine in experimental portal hypertension. Clin Invest Med 1991;14:63-71.

16 Jacob G, Bishara B, Lee SS, et al. Cardiovascular responses to serotonin in experimental liver disease. Hepatology 1991; 14:1235-42.

17 Said O, Bomzon A. The effect of bile duct manipulation and pair-feeding on peripheral vascular neuroeffector mechanisms: In vitro studies. F Pharmacol Toxicol Methods 1995;33:205-12.

18 Krähenbühl S, Talos C, Lauterburg $\mathrm{BH}$, et al. Reduced antioxidative capacity in liver mitochondria from bile duct ligated rats. Hepatology 1995;22:607-12.

19 Gouma DJ, Roughneen PT, Kumar S, et al. Changes in nutritional status associated with obstructive jaundice and biliary drainage in rats. Am F Clin Nutr 1986;44:362-9.

20 Asayama K, Hayashibe H, Dobashi K, et al. Antioxidant enzyme status and lipid peroxidation in various tissues of diabetic and starved rats. Diabetes Res 1989;12:85-91.

21 Shaheen AA, Abd El-Fattah A, Gaz MZ. Effect of various stressors on the level of lipid peroxide, antioxidants and $\mathrm{Na}^{+}, \mathrm{K}^{+}$ATPase activity in rat brain. Experientia 1996;52: 336-9.

22 Lowry $\mathrm{OH}$, Rosebrough NJ, Farr AL, et al. Protein measurement with the Folin phenol reagent. 7 Biol Chem 1951;193:265-75.

23 Adams JD Jr, Lanterberg BH, Mitchell JR. Plasma glutathione and glutathione disulfide in the rat: Regulation and
response to oxidative stress. F Pharm Exp Ther 1983;227: 749-54.

24 Fuhrman B, Oiknine J, Aviram M. Iron induces lipid peroxidation in cultured macrophages, increases their ability to oxidatively modify LDL, and affects their secretory ity to oxidatively modify LDL, and affects

properties. Atherosclerosis 1994;111:65-78.
25 Holley AE, Cheeseman KH. Measuring free radical reactions in vivo. Br Med Bull 1993;49:494-505.

26 El-Saadani M, Esterbauer M, El-Sayed M, et al. A spectrophotometric assay for lipid peroxides in serum lipoproteins using a commercially available reagent. $\mathcal{F}$ Lipid Res 1989;30:627-30

27 Draper HH, Hadley M. Malondialdehyde determination as an index of lipid peroxidation. Methods Enzymol 1990;186: 421-31

28 Gutteridge JMC. Lipid peroxidation and antioxidants as biomarkers of tissue damage. Clin Chem 1995;41:1819-28.

29 Parola M, Leonarduzzi G, Robino G, et al. On the role of lipid peroxidation in the pathogenesis of liver damage induced by long-standing cholestasis. Free Radic Biol Med 1996;20:351-9.

30 Halliwell B, Chirico S. Lipid peroxidation: its mechanism, measurement, and significance. Am f Clin Nutr 1998; 57(suppl):715-25S

31 Kawata S, Chitranukroh A, Owen JS, et al. Membrane lipid changes in erythrocytes, liver and kidney in acute and 
chronic experimental liver disease in rats. Biochim Biophys Acta 1987;896:26-34

32 Kinugasa T, Uchida K, Kadowaki M, et al. The effect of bile duct ligation on bile acid metabolism in rats. 7 Lipid Res 1981;22:201-7.

33 Schroeder ET, Finn AF Jr, Hueber P. Suppression of vascular prostacyclin generation by jaundiced serum: relation to lipid peroxides. F Lab Clin Med 1988;112:784-91.

34 Pratico D, Iuliano L, Basili S, et al. Enhanced lipid peroxidation in hepatic cirrhosis. F Invest Med 1998;46:517.

35 Yamamoto Y, Yamashita S, Fujisawa A, et al. Oxidative stress in patients with hepatitis, cirrhosis, and hepatoma evaluated by plasma antioxidants. Biochem Biophys Res Comm 1998;247:166-70.

36 Poulsen HE, Ranek L, Andreasen PB. The hepatic glutathione content in liver diseases. Scand F Clin Lab Invest 1981; 10ne cont $473-6$

37 Bianchi G, Bugianesi E, Ronchi M, et al. Glutathione kinetics in normal man and in patients with liver cirrhosis. $\mathcal{F}$ ics in normal man and

38 Cheeseman $\mathrm{KH}$, Slater TF. An introduction to free radical biochemistry. Br Med Bull 1993;49:481-93.

39 Berlett BS, Stadtman ER. Protein oxidation in aging, disease, and oxidative stress. F Biol Chem 1997;272:2031316.

40 Henriksen JH, Ring-Larsen H, Christensen NJ. Sympathetic nervous activity in cirrhosis. A survey of plasma catecholamine studies. $\mathcal{F}$ Hepatol $1985 ; 1: 55-65$.

41 Singal PK, Beamish RE, Dhalla NS. Potential oxidative pathways of catecholamines in the formation of lipid peroxides and genesis of heart disease. Adv Exp Med Biol 1983;161:391-5.

42 Laursen JB, Rajagopalan S, Galis Z, et al. Role of superoxide in angiotensin II-induced but not catecholamineinduced hypertension. Circulation 1997;95:588-93.

43 Weiss SJ. Tissue destruction by neutrophils. $N$ Engl f Med 1989;320:365-76.
44 Ostrow JD. Metabolism of bile salts in cholestasis in humans. In: Tavoloni N, Berk PD, eds. Hepatic transport and bile secretion: physiology and pathophysiology. New York: Raven Press, 1993:673-712.

45 Sokol RJ, Winklhofer-Roob BM, Devereaux MW, et al. Generation of hydroperoxides in isolated rat hepatocytes and hepatic mitochondria exposed to hydrophobic bile acids. Gastroenterology 1995;109:1249-56.

46 Ljubuncic P, Fuhrman B, Oiknine J, et al. Effect of deoxycholic acid and ursodeoxycholic acid on lipid peroxidation in cultured macrophages. Gut 1996;39:475-8.

47 Ceryak S, Bouscarel B, Fromm H. Comparative binding of bile acids to serum lipoproteins and albumin. $\mathcal{F}$ Lipid Res 1993;34:1661-74.

48 Sokol RJ, Devereaux M, Khandwala RA. Effect of dietary lipid and vitamin $\mathrm{E}$ on mitochondrial lipid peroxidation and hepatic injury in the bile duct-ligated rat. $\mathcal{F}$ Lipid Res 1991;32:1349-57.

49 Morrow JD, Moore KP, Awad JA, et al. Marked overproduction of non-cyclooxygenase derived prostanoids $\left(\mathrm{F}_{2}-\right.$ isoprostanes) in the hepatorenal syndrome. 7 Lipid Mediat Cell Signal 1993;6:417-20.

50 Fernando B, Marley R, Holt S, et al. $\mathrm{N}$-acetylcysteine prevents development of the hyperdynamic circulation in the portal hypertensive rat. Hepatology 1998;28:689-94.

51 Singh N, Dhalla AK, Seneviratne C, et al. Oxidative stress and heart failure. Mol Cell Biochem 1995;147:77-81.

$52 \mathrm{Ma}$ ZH, Lee SS. Cirrhotic cardiomyopathy: Getting to the heart of the matter. Hepatology 1996;24:451-9.

53 Basile AS, Jones EA. Ammonia and GABA-ergic neurotransmission: Interrelated factors in the pathogenesis of hepatic encephalopathy. Hepatology 1997;25:1303-5.

54 Michalak A, Rose C, Buu PN, et al. Evidence for altered central noradrenergic function in experimental acute liver failure in the rat. Hepatology 1998;27:362-8. 